\title{
VIEWPOINT
}

\section{Initiating breast cancer by PIK3CA mutation}

Todd W Miller*

\begin{abstract}
PIK3CA mutations confer constitutive activation of PI3K, which initiates intracellular kinase signaling cascades that promote cell proliferation and survival. Recent studies by Meyer and colleagues and by Liu and colleagues demonstrate that expression of the H1047R exon 20 mutant of PIK3CA in luminal mammary epithelial cells induces tumorigenesis, implying that PIK3CA mutation is an early event in breast cancer. PIK3CA-H1047R-initiated tumors exhibit variable dependence on the oncogene and variable sensitivity to PI3K inhibition. Amplification of the oncogenes MYC and MET was observed in tumors that recurred following silencing of PIK3CA-H1047R, suggesting that these pathways represent mechanisms of escape from PI3K inhibition.
\end{abstract}

Phosphatidylinositol 3-kinase (PI3K) phosphorylates phosphatidylinositol 4,5-bisphosphate ( $\mathrm{PIP}_{2}$ ) to produce phosphatidylinositol 3,4,5-trisphosphate $\left(\mathrm{PIP}_{3}\right.$ ) at the cytoplasmic face of the plasma membrane. PIP ${ }_{3}$ promotes membrane recruitment and activation of several proteins that drive cell proliferation and survival, including AKT, PDK1, and SGK. Class IA PI3Ks are heterodimers composed of a p110 catalytic subunit and a p85/p55 regulatory subunit, each of which has several isoforms. The PI3K pathway is the most frequently mutated pathway in breast cancer, and mutations occur in signaling nodes both upstream and downstream of PI3K [1].

Activating mutations in PIK3CA (which encodes the p $110 \alpha$ catalytic subunit) occur in approximately $30 \%$ of breast cancers and are more frequent in estrogen receptorpositive $\left(E R^{+}\right)$breast cancers [2,3]. Eighty percent of PIK3CA mutations occur in two 'hot spots' within exons 9 and 20, which encode the helical and kinase domains, respectively. The E542K and E545K (exon 9) mutations

*Correspondence: todd.miller@vanderbilt.edu

Department of Cancer Biology and Breast Cancer Research Program,

Vanderbilt-Ingram Cancer Center, Vanderbilt University School of Medicine, VUMC, 2220 Pierce Avenue, 771 PRB, Nashville, TN 37232, USA may confer a gain-of-function by disrupting an inhibitory interaction between $\mathrm{p} 110 \alpha$ and $\mathrm{p} 85$ [4]. The H1047R (exon 20) mutation may induce an allosteric change that mimics Ras-GTP binding, making this mutant independent of interaction with Ras-GTP [5]. Both mutants are constitutively active, transform cells in culture, and promote tumorigenicity in xenograft models. Cancer cell lines harboring PIK3CA mutations are highly sensitive to PI3K pathway inhibitors [6,7], rendering this pathway a drug target of high interest for cancer therapy. PIK3CA mutations have been found at similar frequencies in breast ductal carcinoma in situ (DCIS) lesions, DCIS adjacent to invasive ductal carcinoma (IDC), and IDC [8], suggesting that these mutations are early events in breast tumorigenesis and therefore may promote transformation of normal breast epithelial cells.

A recent study by Meyer and colleagues [9] revealed that expression of the PIK3CA-H1047R mutant in mammary epithelial cells is sufficient to induce tumor formation in transgenic mice. PIK3CA-H1047R expression driven by Cre-mediated recombination induced by either the WAP promoter (which is active in alveolar progenitor and differentiated secretory luminal epithelial cells) or the $M M T V$ promoter (which is active in differentiated luminal mammary epithelial cells) induced the formation of mammary tumors of varying histologic subtypes. Tumor cells expressed markers associated with both luminal and basal epithelial lineages, suggesting that tumors with basal characteristics can arise from luminal cells. The authors postulate that PIK3CA-H1047R may (a) transform multi-potent progenitor cells to allow both luminal and basal differentiation, (b) induce de-differentiation of luminal cells to multi-potent progenitors, which then give rise to both lineages, or (c) do both. Involuting mammary glands (which undergo ductal pruning following pregnancy and lactation) from PIK3CA-H1047R mice showed a reduction in the number of apoptotic cells and delayed involution in comparison with controls. PIK3CAH1047R tumors also showed very low rates of apoptosis and higher levels of phosphorylated AKT than mammary tumors from another model (MMTV-NeuNT), suggesting that PIK3CA-H1047R prevents cell death by increased PI3K/AKT pathway activation.

In another study, Liu and colleagues [10] reported that PIK3CA-H1047R-induced mammary tumors exhibit 
variable dependence on this oncogene. Transgenic mice expressed PIK3CA-H1047R under the control of an MMTV-regulated, doxycycline-inducible system. Mice treated with doxycycline showed increased phospho-AKT levels in mammary epithelial cells and formed mammary tumors of varying histologic subtypes. Silencing of PIK3CA-H1047R by withdrawal of doxycycline decreased tumor phospho-AKT levels, decreased proliferation, increased apoptosis, and induced complete tumor regression in one third of the mice. Two thirds of tumors partially regressed and then resumed growth. Some recurrent tumors that maintained high levels of P-AKT and P-S6 were sensitive to the PI3K inhibitor GDC-0941, whereas tumors with low P-AKT and P-S6 were insensitive to this agent. This suggests that some PIK3CAH1047R-induced tumors escape from dependence on PI3K. GDC-0941-resistant and PIK3CA-H1047R-independent tumors exhibited amplification of the oncogenes $M Y C, M D M 2$, and/or MET. The authors demonstrated tumor dependence on $M Y C$ (using short-hairpin RNA knockdown) and $M E T$ (using a kinase inhibitor) and showed that MYC overexpression circumvented dependence on PI3K.

These studies have important implications for the role of PI3K mutations in breast cancer. First, these works show that PIK3CA-H1047R induces mammary epithelial cell transformation in vivo and support the notion that PIK3CA mutation is an early event in breast cancer. Second, the paper by Liu and colleagues [10] affirms that PIK3CA-mutant tumors are dependent, in whole or in part, on this oncogene. Some tumors that recurred following silencing of PIK3CA-H1047R showed sensitivity to a PI3K inhibitor, indicating continued addiction to PI3K. Since PI3K pathway inhibitors preferentially inhibit the growth of cancer cells harboring PIK3CA mutations [6,7], such mutations are being used as an inclusion criterion in ongoing clinical trials with these agents to enroll patients who are most likely to benefit. The authors' findings support this concept. Third, in agreement with the existence of PIK3CA mutations in all subtypes of breast cancer, PIK3CA-H1047R expression induced mouse mammary tumors expressing both luminal and basal markers. Issues that remain to be addressed are the mechanism underlying the variable histologic subtypes of PIK3CA-H1047R-induced mammary tumors and the effects of PIK3CA-H1047R on luminal/ basal cell differentiation. Fourth, the majority of PIK3CAH1047R-induced mammary tumors recurred following an initial regression after oncogene silencing. Such recurrence was driven by $M Y C$ and $M E T$, suggesting that therapies targeting the PI3K pathway may be most effective when used in combination with agents that block such escape mechanisms.

\section{Abbreviations}

DCIS, ductal carcinoma in situ; IDC, invasive ductal carcinoma; PI3K, phosphatidylinositol 3-kinase; PIP $3^{\prime}$ phosphatidylinositol 3,4,5-trisphosphate.

Competing interests

The author declares that he has no competing interests.

\section{Acknowledgments}

This work is supported by National Cancer Institute grants K99CA142899 (TWM), Breast Cancer Specialized Program of Research Excellence (SPORE) grant P50CA98131, and Vanderbilt-Ingram Cancer Center Support Grant P30CA68485.

Published: 7 February 2012

\section{References}

1. Miller TW, Rexer BN, Garrett JT, Arteaga CL: Mutations in the phosphatidylinositol 3-kinase pathway: role in tumor progression and therapeutic implications in breast cancer. Breast Cancer Res 2011, 13:224.

2. Liu P, Cheng H, Roberts TM, Zhao JJ: Targeting the phosphoinositide 3-kinase pathway in cancer. Nat Rev Drug Discov 2009, 8:627-644.

3. Saal LH, Holm K, Maurer M, Memeo L, Su T, Wang X, Yu JS, Malmström PO, Mansukhani M, Enoksson J, Hibshoosh H, Borg A, Parsons R: PIK3CA mutations correlate with hormone receptors, node metastasis, and ERBB2, and are mutually exclusive with PTEN loss in human breast carcinoma. Cancer Res 2005, 65:2554-2559.

4. Miled N, Yan Y, Hon WC, Perisic O, Zvelebil M, Inbar Y, Schneidman-Duhovny D, Wolfson HJ, Backer JM, Williams RL: Mechanism of two classes of cancer mutations in the phosphoinositide 3-kinase catalytic subunit. Science 2007, 317:239-242.

5. Zhao L, Vogt PK: Helical domain and kinase domain mutations in p110alpha of phosphatidylinositol 3-kinase induce gain of function by different mechanisms. Proc Natl Acad Sci U S A 2008, 105:2652-2657.

6. Maira SM, Pecchi S, Huang A, Burger M, Knapp M, Sterker D, Schnell C, Guthy D, Nagel T, Wiesmann M, Brachmann SM, Fritsch C, Dorsch M, Chene P, Shoemaker K, De Pover A, Menezes D, Martiny-Baron G, Fabbro D, Wilson C, Schlegel R, Hofmann F, Garcia-Echeverria C, Sellers WR, Voliva CF: Identification and characterization of NVP-BKM120, an orally available pan class I PI3-Kinase inhibitor. Mol Cancer Ther 2011 Dec 21. [Epub ahead of print].

7. She QB, Chandarlapaty S, Ye Q, Lobo J, Haskell KM, Leander KR, DeFeo-Jones D, Huber HE, Rosen N: Breast tumor cells with PI3K mutation or HER2 amplification are selectively addicted to Akt signaling. PLOS ONE 2008, 3:e3065.

8. Miron A, Varadi M, Carrasco D, Li H, Luongo L, Kim HJ, Park SY, Cho EY, Lewis G, Kehoe S, Iglehart JD, Dillon D, Allred DC, Macconaill L, Gelman R, Polyak K: PIK3CA mutations in in situ and invasive breast carcinomas. Cancer Res 2010, 70:5674-5678.

9. Meyer DS, Brinkhaus H, Muller U, Muller M, Cardiff RD, Bentires-Alj M: Luminal expression of PIK3CA mutant H1047R in the mammary gland induces heterogeneous tumors. Cancer Res 2011, 71:4344-4351.

10. Liu P, Cheng H, Santiago S, Raeder M, Zhang F, Isabella A, Yang J, Semaan DJ, Chen C, Fox EA, Gray NS, Monahan J, Schlegel R, Beroukhim R, Mills GB, Zhao JJ: Oncogenic PIK3CA-driven mammary tumors frequently recur via PI3K pathway-dependent and PI3K pathway-independent mechanisms. Nat Med 2011, 17:1116-1120.

doi: $10.1186 /$ bcr3103

Cite this article as: Miller TW: Initiating breast cancer by PIK3CA mutation. Breast Cancer Research 2012, 14:301. 